\title{
Retrieval of memory in the pigeon by context manipulations
}

\author{
DAVID R. THOMAS and ALAN R. McKELVIE \\ University of Colorado, Boulder, Colorado 80309
}

\begin{abstract}
In Experiment 1, 12 pigeons were given eight sessions of VI single stimulus training with a color in a particular context followed by eight sessions of similar training with a line angle in another context. On the next day, half of the subjects were tested for wavelength and angularity generalization in each of the two contexts, a procedure that was thus consistent with training for one dimension and inconsistent for the other. The subjects made significantly more responses to each training stimulus under the consistent context condition, but there was no difference in absolute or relative generalization slopes. In Experiment 2,12 pigeons were trained as in Experiment 1, but during generalization testing they were exposed to both contexts sequentially. Under the consistent context condition, the subjects responded more to the two training stimuli and yielded sharper absolute and relative wavelength generalization gradients: Under the inconsistent context condition, responding to the training wavelength was substantially disrupted. Thus, under appropriate testing conditions, contextual control over both the amount and the selectivity of responding can be demonstrated.
\end{abstract}

Spear $(1971,1978)$ has proposed that success at retrieval of a memory is a function of the extent to which the subject notices, during retention testing, ambient contextual stimuli that were present but inconsequential to the target learning task and were stored as attributes of the target memory. The role of contextual retrieval cues in human memory has frequently been demonstrated. Rand and Wapner (1967) reported that the retention of a list of nonsense syllables was better when the subject was tested in the same postural position as during original learning (i.e., standing up or lying down). Greenspoon and Ranyard (1957) found that the color and location of the experimental room functioned as retrieval cues in a similar manner.

A typical example from the animal memory literature is a proactive interference (PI) experiment reported by Spear (1971). Rats trained on a passive avoidance task followed by an active avoidance task typically showed recency in a delayed test, behaving in accordance with the active avoidance contingency. If a tone had been present during passive avoidance training and was present in the subsequent retention test, however, then behavior more appropriate to the passive avoidance task was exhibited in the retention test.

In our laboratory, we (Thomas, McKelvie, Ranney, \& Moye, 1981) recently completed a study conceptually similar to the Spear (1971) experiment. Pigeons

Requests for reprints should be addressed to David R. Thomas, Department of Psychology, Campus Box 345, University of Colorado at Boulder, Boulder, Colorado 80309. This research was supported by NSF Research Grant BNS-7801407. It was reported by Alan McKelvie at the April 1981 meetings of the Rocky Mountain Psychological Association in Denver. first learned a successive wavelength discrimination, $538 \mathrm{~nm} \mathrm{~S}+$ (reinforced) vs. $576 \mathrm{~nm} \mathrm{~S}$ - (extinguished), followed by a reversal of this discrimination (i.e., $576 \mathrm{~nm} \mathrm{S+,} 538 \mathrm{~nm} \mathrm{S-).} \mathrm{For} \mathrm{a} \mathrm{control} \mathrm{group,} \mathrm{a}$ generalization test administered in extinction a day later revealed recency (i.e., the gradients all peaked at the Problem $2 \mathrm{~S}+$. For one experimental group, however, as in Spear's (1971) experiment, Problem 1 had been learned in a distinctive context (Context 1 , either houselight + white noise or no houselight + $1,000-\mathrm{Hz}$ tone), and generalization testing was carried out in this context. The result was substantial PI; no gradients showed recency. Some peaked at the Problem $1 \mathrm{~S}+$, but most showed peaks at both $\mathrm{S}+$ values or a peak intermediate between them. Thus, Context 1 was a sufficiently effective retrieval cue to prevent retrieval of Problem 2 but not, in most cases, to permit successful retrieval of Problem 1. A second experiment, in which subjects were tested alternately in the contexts in which the two problems had been learned, demonstrated that successful retrieval of the memory of both problems was possible. Under this test condition, each subject yielded a gradient appropriate to the problem learned in each context (i.e., the gradient peaked at $538 \mathrm{~nm}$ in Context 1 and at $576 \mathrm{~nm}$ in Context 2).

The degree of control over behavior by context in Thomas et al.'s (1981) Experiment 2 was quite striking, resembling that which would have been expected had the subjects been trained on an explicit conditional discrimination with the context as the superordinate cue. Note, however, that such conditional discriminations are usually difficult to establish in pigeons when traditional methods involving repeated alternations of the conditional (superordinate) stimuli 
are employed (cf. Richards, 1979). In the Thomas et al. experiment, there was only one alternation in training between the two contexts; furthermore, the association between Context 2 and Problem 2 was formed in a single training session. It is of interest to determine which aspect of Thomas et al.'s procedure accounted for the success of the retrieval process in their experiment. One possible factor was their use of a reversal paradigm. Perhaps the "surprise" (Kamin, 1969) of the reversal manipulation called attention to the change in contextual conditions, thereby increasing their significance. It is also possible that the use of discrimination training in the Thomas et al. study was a critical factor. Thomas (1970) has argued that discrimination training enhances attention to cues, such as the context, that are inconsequential to the discrimination problem itself.

In the Thomas et al. (1981) study, the success of retrieval was reflected primarily in the obtaining of a generalization gradient with a peak of responding at the appropriate $S+$ value. In the present experiments, single stimulus training rather than discrimination training was used. Thomas and Lopez (1962) showed that relative generalization gradients obtained $24 \mathrm{~h}$ after the completion of single stimulus training were reliably flatter than those obtained in an immediate (i.e., 1-min-delayed) test. They attributed this flattening to some forgetting of the value of the training stimulus. Interestingly, there was no reliable reduction in number of responses to the training stimulus over the $24-\mathrm{h}$ period in their experiment. This finding may merely indicate that generalization slope is a more sensitive index of forgetting than is absolute response strength to the training stimulus. However, Newlin and Thomas (in press) have argued that the two measures reflect different processes (i.e., those of dimensional stimulus control and excitatory stimulus control, respectively). As evidence for this position, Newlin and Thomas presented data from several experiments indicating that the two measures may vary in opposite directions with manipulations of the same independent variable (e.g., brief vs. continuous stimulus exposures). The present experiments enabled us to study the way in which measures of excitatory and dimensional stimulus control are affected by the same retrieval cue manipulations. Since forgetting following single stimulus training is reflected in a flattening of the generalization gradient and (in some cases) in a reduction of responding to the training stimulus, successful retrieval of the memory of training should be reflected in changes in the opposite direction (i.e., a sharpening of generalization and an increase in responding to the training stimulus).

There are several reasons why relative generalization slope has become the typical measure of dimensional stimulus control. The transformation of each subject's gradient into a percentage of total responses equates the contribution of each subject to the group average. It also eliminates the large individual differences in level of responding, which often make statistical significance of differences in generalization slope difficult to demonstrate. It is generally the case that absolute and relative gradients reveal parallel effects of a given experimental manipulation. For example, both are sharpened by discrimination training and flattened by nondifferential training (cf. Thomas, 1970). This parallel is not always present, however. Thomas and King (1959) reported a deprivation level study in which, within each deprivation condition, the high-responding animals gave relative gradients that were indistinguishable from those of the low responders. Their absolute gradients were, of course, substantially sharper. Given the possibility that the context manipulation might affect absolute and relative gradients differently, both $a b-$ solute and relative gradients will be reported and discussed in this paper.

\section{EXPERIMENT 1}

In Experiment 1, pigeons were given single stimulus training to peck at a green keylight for variable interval reinforcement in a particular context. They then received single stimulus training with a horizontal line stimulus in a different context. For parposes of retention testing, the birds were divided into two groups, one tested in Context 1 and one tested in Context 2 . The generalization tests were conducted, in extinction, 1 day after the completion of training, and each test included stimuli from both wavelength and line-orientation dimensions. Thus, for each bird the test context was consistent with one of the two training problems and inconsistent with the other. The experimental design was within subjects with regard to the assessment of memory for the two training problems, but between subjects with regard to the use of a single test context with each bird. Testing with both dimensions provides a control for any nonassociative effects of the contextual cues (e.g., masking) because response strength should be greater and the generalization gradient should be sharper only when testing is carried out in the consistent context condition. If any particular set of contextual stimuli were more effective in retrieving the memory of its associated training or, on the other hand, were more effective in masking the expression of stimulus control, such effects would be revealed by the counterbalancing of contextual stimuli across conditions, as described below. Presumably, what is critical is the relationship between each context and its associated problem, rather than the context per se.

\section{Method}

Subjects. The subjects were 12 experimentally naive pigeons obtained from a local supplier and maintained at $75 \%$ of their ad-lib weights. They were housed in individual cages in a colony 
room with a 16/8-h light/dark cycle. Training and testing sessions commenced $8 \mathrm{~h}$ after the onset of the light cycle.

Apparatus. Four similar sound-attenuating ice chests with interior dimensions of $31 \times 35 \times 36 \mathrm{~cm}$ served as experimental chambers. Each chamber had a wire-mesh floor, and one wall constituted an intelligence panel constructed of aluminum with a 2.2-cm-diam translucent plastic response key centered $20 \mathrm{~cm}$ above the floor. Approximately $13 \mathrm{~cm}$ directly below the key, a $4.5 \times 6 \mathrm{~cm}$ aperture allowed access to mixed grain from a solenoid-operated hopper. Behind the front wall and directly to the right of the aperture was a $10-\mathrm{cm}$-diam speaker.

Stimuli were projected onto a translucent screen on the front of IEE (Series 0010) in-line display cells mounted $.635 \mathrm{~cm}$ behind the key aperture. Display cells fitted with Kodak Wratten filters 75 , $74,99,73$, and $72 b$ provided chromatic stimuli with nominal peak wavelength transmission of $490,538,555,576$, and $606 \mathrm{~nm}$, respectively. The display cell allowed the projection of a $.2-\mathrm{cm}$-wide white line on a black surround with angular orientations of 0 (horizontal), 15, 30,45, and $60 \mathrm{deg}$. Projection of these stimuli was accomplished by illuminating miniature 14-V lamps (G.E. No. 1815). Chamber illumination was provided by lighting a single G.E. No. 1829 lamp located behind a $3 \times 31 \mathrm{~cm}$ strip of diffusing Plexiglas that extended across the chamber above the intelligence panel. The only other source of illumination in the chambers was provided by an ESB 24-V lamp that was above the food hopper and was illuminated during the hopper presentation.

A standard electronic tone generator provided white noise or a 1,000- $\mathrm{Hz}$ tone at $80 \mathrm{~dB}$ SPL (re: $.0002 \mathrm{dyn} / \mathrm{cm}^{2}$ ). Exhaust fans provided masking noise. Reinforcement was $3 \mathrm{sec}$ of access to the food hopper filled with mixed grain. All recording and control were accomplished with standard relay circuitry in an adjoining room.

Procedure. In training, subjects were treated identically except for the ambient contextual stimuli present. For six subjects, the first context consisted of houselight-on plus a 1,000-Hz tone (HLT); for the other six subjects, the first context was houselight-off plus white noise ( $\overline{\mathrm{HLN}})$. All subjects were magazine trained and hand shaped to peck the 555-nm keylight and were allowed to earn 60 reinforcers on a gradually increasing variable interval (VI) schedule. On Day 2, subjects were given single stimulus training with the $555-\mathrm{nm}$ keylight in their appropriate contexts to a criterion of 40 reinforcers earned on a VI $60-\mathrm{sec}$ schedule. Thereafter, subjects were run daily in 30 -min sessions of VI $60-\mathrm{sec}$ training consisting of 30 trials of $55-\mathrm{sec}$ duration separated by $5-\mathrm{sec}$ blackouts. After eight sessions of wavelength training, all subjects received eight sessions of training with the 0-deg line under the alternate context condition. For each subject, the context associated with the wavelength training will be designated Context 1 , and that associated with the line angle training will be designated Context 2 .

Generalization tests were administered, in extinction, $24 \mathrm{~h}$ after the last training session. The test included the five wavelengths $490,538,555,576$, and $606 \mathrm{~nm}$ and the five line angles 0,15 , 30,45 , and 60 deg. The test involved a random arrangement of the five stimuli within each dimension into blocks of 10 stimuli, with each subject exposed to a block of wavelengths, then a block of lines, then wavelengths, and so on, for a total of three blocks of each dimension. During the test, stimulus presentations were of 30-sec duration separated by 5-sec blackouts. Half of the subjects were tested in Context 1 and half in Context 2 . Within each of these groups, three subjects were tested in the HLT condition and three in the $\overline{\mathrm{HL}} \mathrm{N}$ condition.

\section{Results and Discussion}

Figure 1 presents the mean generalization gradients. The curves in the upper panels are the absolute generalization gradients; the relative generalization gradients are in the lower panels. The wavelength gradients appear in the left panels, while the angularity gradients are in the right panels. The data were pooled

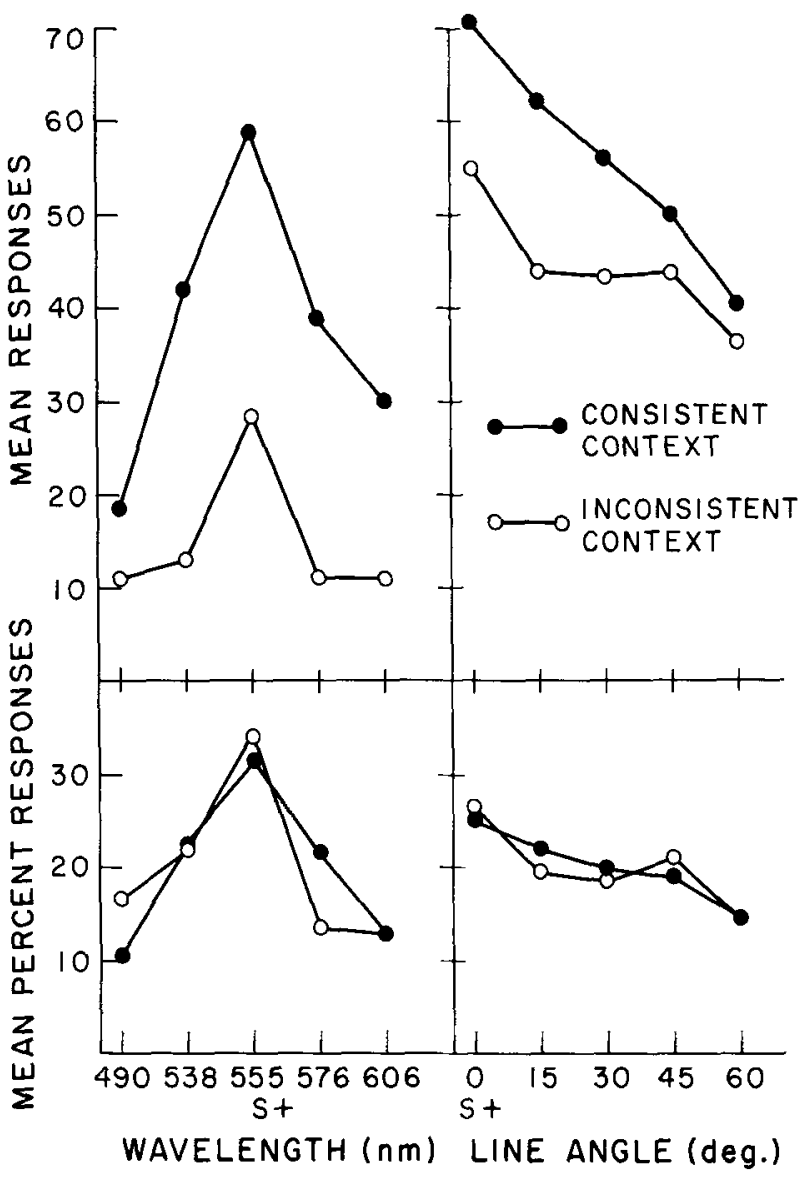

Figure 1. Absolute and relative generalization gradients obtained in Experiment 1, averaged across three blocks of testing (see text for details).

across contextual stimuli (HLT and $\overline{\mathrm{HL}} \mathrm{N}$ ) since these had no effect. Absolute and relative generalization gradients were calculated for each block and then averaged to minimize the effects of extinction on the relative gradients. The absolute gradients in the top panels of Figure 1 suggest that the context condition, consistent vs. inconsistent, had an effect. For both dimensions, the gradient was higher and sharper under the consistent context condition.

Let us consider first the measure of excitatory stimulus control (i.e., the number of responses to the training stimuli). The mean number of responses to $555 \mathrm{~nm}$ was 59 in Context 1 (consistent) vs. 26 in Context 2; for the 0 -deg line, the corresponding values were 55 in Context 1 (inconsistent) vs. 72 in Context 2. A mixed-design analysis of variance (group $\times$ test dimension) performed on the number of responses to the $\mathrm{S}+$ indicated that this difference, in favor of the consistent context condition, was statistically significant $[F(1,10)=5.12, \mathrm{p}<.05]$.

The upper panels of Figure 1 suggest that dimensional stimulus control (i.e., the slope of the absolute generalization gradients) also differed with respect to context. However, analysis of variance disconfirmed this result. Separate mixed-design ANOVAs (group 
$X$ stimulus) performed on the responses obtained during the wavelength and line angle tests yielded no group $X$ stimulus interactions $[F s(4,40)=1.57$ and 1.56 for wavelength and line angle, respectively; ps $=.2]$. As is typical in free-operant experiments, individual variation in response levels under each condition was so great that the differences apparent in the figure did not achieve statistical significance.

To the extent that there is some difference, the results suggest that the consistent context condition acts as a constant multiplier of responding to all stimulus values. This relationship is made clear in the lower panels of Figure 1, in which it can be seen that for each dimension the relative generalization gradients obtained under consistent and inconsistent context conditions are virtually superimposed. Clearly, the results indicate that Context 1 was associated with the wavelength problem and Context 2 was associated with the line angle problem. The memory of training was retrieved more effectively in the consistent than in the inconsistent context, but the effects are seen only in the excitatory measure (i.e., absolute response level).

\section{EXPERIMENT 2}

In the Thomas et al. (1981) study, it was shown that the effectiveness of the context as a retrieval cue could be substantially enhanced by the use of a generalization test procedure in which both contexts were presented sequentially. Experiment 2 was designed to determine whether a similar result would be obtained in the experimental paradigm used here.

\section{Method}

Subjects. The subjects were 12 experimentally naive pigeons maintained as in Experiment 1.

Apparatus. The apparatus was the same as that in Experiment 1.

Procedure. The training procedure was the same as that in Experiment 1. Again, for half of the subjects Context 1 (associated with wavelength) was HLT, and for half it was HLN. The conditions for Context 2 were similarly counterbalanced.

Generalization testing in extinction was carried out $24 \mathrm{~h}$ after the last training session. Test stimuli were presented for $30 \mathrm{sec}$, separated by 5 -sec blackouts. For all subjects, testing began with a block of 10 wavelengths, followed by a block of 10 line angles, followed by a block of wavelengths, and so on. The context was changed after every two test blocks, and the test started with Context 1 for half of the subjects and with Context 2 for the other half. Testing continued until each subject experienced three blocks from each dimension in each context condition (i.e., a total of 12 blocks).

\section{Results and Discussion}

Figure 2 presents the mean generalization gradients obtained in this experiment. The curves in the upper panels are the absolute generalization gradients; the relative generalization gradients are in the lower panels. The wavelength generalization gradients appear in the left panels, while the angularity gradients are in the right panels. The data were pooled across con-

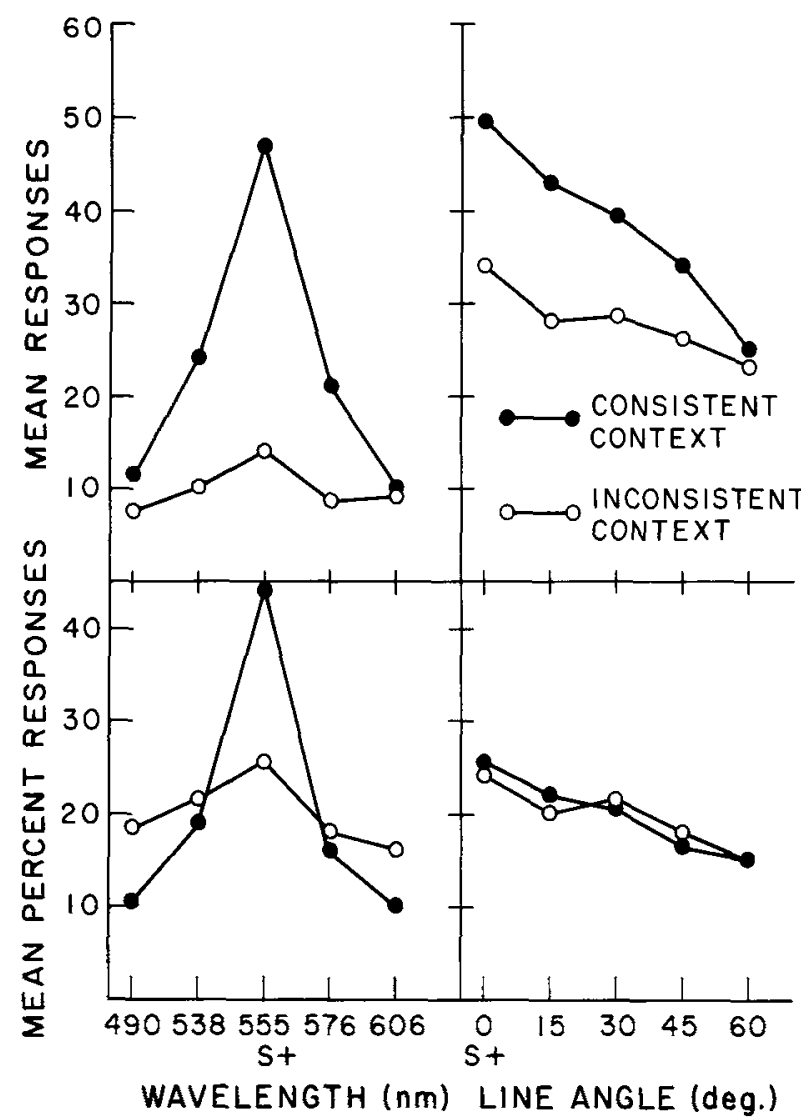

Figure 2. Absolute and relative generalization gradients obtuined in Experiment 2, averaged across three blocks of testing (see text for details).

textual stimuli (HLT and $\overline{\mathrm{HLN}}$ ) since these had no effect. Absolute and relative generalization gradients were calculated for each block and averaged across test blocks. As in Experiment 1, test context had a significant effect on level of responding to the training stimuli. The mean number of responses to $555 \mathrm{~nm}$ was 47 in Context 1 vs. 14 in Context 2; for the 0 -deg line, the corresponding values were 34 in Context 1 vs. 50 in Context 2. A two-way repeated measures ANOVA (context $\times$ test dimension) performed on the responses to $S+$ indicated that significantly more responding occurred to the training stimulus in the consistent than in the inconsistent condition [context main effect: $F(1,11)=40.2, p<.01]$. It is clear from Figure 2 that the use of a consistent context resulted in both higher and sharper absolute generalization gradients. In contrast with the results of Experiment 1, the two-way repeated measure ANOVAs (context $x$ stimuli) performed separately for each dimension showed that the absolute gradient slopes of the consistent vs. inconsistent conditions were significantly different $[\mathrm{Fs}(4,44)=12.9$ and 4.4 for wavelength and line angle, respectively; ps $<.01$ ]. Indeed, responding to the wavelengths under the inconsistent context condition was severely disrupted; it was low, sporadic, and largely unsystematic. 
The lower panels of Figure 2 indicate that the effectiveness of context as a retrieval cue was greater for the colors than for the lines. Again, the effect of context on angularity gradients was multiplicative, so the relative gradients are superimposed. In the case of wavelength, however, there was a substantial difference in the slope of the relative generalization gradients. A two-way repeated measures ANOVA (context $x$ stimuli) indicated that the gradient obtained under the consistent context condition was significantly sharper than that obtained under the inconsistent context condition [context $\times$ stimuli interaction: $F(4,44)=6.9$, $\mathrm{p}<.001]$.

In general, the effect of the context condition was greater in Experiment 2, in which subjects were exposed to both contexts during a test session, than it had been in Experiment 1, in which testing was carried out under a single context condition. Furthermore, in Experiment 2 the effect of the context was much greater with the wavelength than with the line angle stimuli. There are several possible reasons for this. No attempt had been made to equate the two sets of test stimuli for discriminability, and the gradients along the angularity dimension were rather flat under all conditions. Thus, there was little room to show further flattening. Furthermore, at the time of the test, the subjects had seen the 0 -deg line on the preceding day but had not seen the $555-\mathrm{nm}$ wavelength value for 9 days. It seems reasonable that reminder cues would be more effective under the latter condition. ${ }^{1}$

Again, as in Experiment 1, the measures of excitatory and dimensional stimulus control were in agreement in indicating that retrieval of the memory for training was more successful under the consistent context condition. This time, however, both the response strength and the absolute generalization slope differences were significant.

The finding that retention performance is better in the consistent context than in the inconsistent context can be interpreted in several different ways. The consistent context may facilitate retrieval, the inconsistent context may interfere with retrieval, or both effects may occur. To determine which of these possibilities occurred, a "neutral" context is required for comparison purposes, but the definition of what constitutes a neutral context presents a logically formidable problem. A novel context is unsuitable because it leads to a disruption of operant keypecking, thus providing no measure of dimensional stimulus control. An alternative definition of a neutral context is that it is a context that is demonstrated to be ineffective. Thus, with regard to the measure of relative generalization slope, both contexts in Experiment 1 meet this definition. The best estimate of the measure of relative generalization slope under a neutral context condition is obtained by pooling the measures obtained under the two (ineffective) context condi- tions of Experiment 1. For wavelength, the resultant mean is $33 \%$ of total responses to $555 \mathrm{~nm}$. This value falls between the values of $43 \%$ for the consistent context condition and $26 \%$ for the inconsistent context condition in Experiment 2. Although it is admittedly speculative, we would suggest, based upon this comparison, that under the conditions of Experiment 2, Context 1 facilitated the retrieval of the memory of the training wavelength, whereas Context 2 interfered with the retrieval of this memory. ${ }^{2}$

The effect of the consistent context in facilitating the retrieval of memory was anticipated, based on the results of our previous research. We did not expect, however, the substantial interference we observed in the inconsistent context condition. Many of the individual subjects' gradients obtained under this condition were essentially flat or peaked at a nontraining value, suggesting an inhibitory process. Note that, unlike the Thomas et al. (1981) study, in which a reversal was used, the two "competing" memories in this study were orthogonal (i.e., peck at a color, and peck at a line). Thus, there is no clear-cut basis for interference between them. Why the presence during testing of a retrieval cue for one of the memories should so completely disrupt expression of the memory associated with the alternative cue (under the condition in which both cues are experienced within the session) is unclear at present. Perhaps a useful way to conceptualize the effect is that the inconsistent context renders the memory of the target problem inaccessible in its presence (cf. Tulving \& Pearlstone, 1966, for a discussion of the distinction between availability and accessibility in human verbal memory).

It is important to note that a critical determinant of the effectiveness of context as a retrieval cue is the procedure employed-specifically, whether the retrieval cue for one memory or the cues for both (potentially conflicting) memories are used within a session. Given that the subject has the opportunity to compare different retrieval cues during testing, neither a reversal paradigm nor the use of discrimination training is required to produce evidence of very substantial control over behavior by ambient contextual stimuli used as retrieval cues.

It may be appropriate in closing to raise the question of whether the results of these experiments require the postulation of a construct of memory for their interpretation. Taken alone, the results of Experiment 1 do not. Butter (1963) trained pigeons to peck at a vertical line of a particular color and then varied both the angle and the color in generalization testing. In the presence of a nonvertical line, the wavelength gradient was multiplicatively flattened, a finding parallel to that produced by the inconsistent context in Experiment 1. The differences between the results of our two experiments, however, seem to require the constructs of memory and its retrieval. Note that training was identical in the two experiments; thus, 
differences in the success with which subjects manifested their learning in test performance lend themselves to an interpretation postulating a retrieval process.

\section{REFERENCES}

ButTe R, C. M. Stimulus generalization along one and two dimensions in pigeons. Journal of Experimental Psychology, 1963, 65, 339-346.

Greenspoon, J., \& Ranyard, R. Stimulus conditions and retroactive inhibition. Journal of Experimental Psychology, 1957, $53,55-59$.

Kamin, L. J. Predictability, surprise, attention and conditioning. In B. Campbell \& R. Church (Eds.), Punishment and aversive behavior. New York: Appleton-Century-Crofts, 1969.

Newlin, R. J., \& Tномas, D. R. On the acquisition and measurement of stimulus control in pigeons. Animal Learning \& Behavior, 1982, in press.

RAND, G., \& Wapner, S. Postural status as a factor in memory. Journal of Verbal Learning and Verbal Behavior, 1967, 6, 268-271.

RICHARDS, R. W. Stimulus control following training on a conditional discrimination. Animal Learning \& Behavior, 1979, 17, 309-312.

Spear, N. E. Forgetting as retrieval failure. In W. K. Honig \& P. H. R. James (Eds.), Animal memory. New York: Academic Press, 1971.

Spear, N. E. The processing of memories: Forgetting and retention. Hiilsdale, N.J: Erlbaum, 1978.

Thomas, D. R. Stimulus selection, attention, and related matters. In J. H. Reynierse (Ed.), Current issues in animal learning. Lincoln: University of Nebraska Press, 1970.
Thом AS, D. R., \& KING, R. A. Stimulus generalization as a function of the level of motivation. Journal of Experimental Psychology, 1959, 57, 323-328.

Thomas, D. R., \& Lopez, L. J. The effect of delayed testing on generalization slope. Journal of Comparative and Physiological Psychology, 1962, 44, 541-544.

Thomas, D. R., McKelvie, A. R., Ranney, M., \& Moye, T. B. Interference in pigeons' long-term memory viewed as a retrieval problem. Animal Learning \& Behavior, 1981, 9, 581 -586.

Tulving, E., \& Pearlstone, Z. Availability vs. accessibility of information in memory for words. Journal of Verbal Learning and Verbal Behavior, 1966, 5, 381-391.

\section{NOTES}

1. Note that Thomas and Lopez (1962) had found that generalization gradients obtained after a 1-week delay were no flatter than those obtained after a 1-day delay. This may reflect a floor effect in the generalization measure, rather than the absence of any further forgetting. The "retrievability" of a memory may provide an alternative index of the extent to which it had been forgotten in the first place.

2. Note that all subjects in both experiments were trained by the same experimenter, in the same apparatus, under identical procedures, and during the same time period. In some sense, then, the cross-experiment comparison may be viewed as a comparison between groups in the same experiment. The groups differed only in test length and test procedure, which is precisely the variable of major importance in this study.

(Manuscript received July 28, 1981; revision accepted for publication October $27,1981$. 